\title{
La Red de Registros de Cáncer con Base Poblacional en México
}

\author{
Alejandra Palafox-Parrilla ${ }^{1}$ y Alejandro Mohar-Betancourt ${ }^{1,2^{*}}$ \\ ${ }^{1}$ Instituto Nacional de Cancerología, Unidad de Epidemiología; ${ }^{2}$ Comisión Coordinadora de Institutos Nacionales de Salud y Hospitales de Alta Especialidad. Ciudad de \\ México, México
}

Se estima que en nuestro país se diagnostican 200,000 nuevos casos de cáncer por año ${ }^{1}$. El impacto económico de este grupo de enfermedades incluye tanto la pérdida de ingresos como los gastos asociados a su tratamiento y control. La carga económica que genera el cáncer es evidente en los costos de su diagnóstico, atención médica, seguimiento y, en muchos casos, cuidados paliativos. La Organización Mundial de la Salud establece que el cáncer es la segunda causa de muerte en el mundo; en 2015 ocasionó 8.8 millones de defunciones ${ }^{1}$. Casi una de cada seis defunciones en el mundo se debe a este grupo de enfermedades. Cerca del $70 \%$ de las muertes por cáncer se registran en países de ingresos medios y bajos y, solo uno de cinco de este grupo de países dispone de los datos epidemiológicos por medio de Registros de Cáncer como instrumento para impulsar políticas de lucha y control ${ }^{1}$.

\section{REGISTROS DE CÁNCER EN AMÉRICA}

A partir de 1900 surgieron proyectos individuales e institucionales para el estudio del cáncer mediante su identificación y registro. Es en 1935 cuando el primer Registro de Cáncer de Base Poblacional (RCBP) se estableció en el Estado de Connecticut (EE.UU.), que derivó en el National Cancer Data Base y en el SurveiIlance, Epidemiology and End Results Program ${ }^{2,3}$.

\section{REGISTROS DE CÁNCER EN AMÉRICA LATINA}

Los tres primeros Registros en América Latina fueron el de Puerto Rico en 1950, seguido por el de Cali, Colombia, en 1962, y el de Sao Paulo, Brasil, en 1963. Cabe señalar que solo del 6 al 7\% de la población está cubierta por registros poblacionales, en contraste con el 83\% en América del Norte y el 32\% en Europa ${ }^{4}$.

El valor innegable de los Registros de Cáncer de Base Poblacional es que son el instrumento idóneo para impulsar políticas públicas de control de cáncer en diversas comunidades. Son sistemas de vigilancia epidemiológica activa y centinela de la enfermedad, que proporcionan un panorama más fidedigno del cáncer en un área geográfica determinada. Por lo tanto, tienen un papel fundamental en la planificación y evaluación de los programas de prevención y control de la enfermedad. En condiciones ideales, es posible obtener un panorama de las tendencias de la enfermedad a partir de los datos concernientes a la incidencia por unidad de tiempo. Sin embargo la validez de estos datos solo se puede y debe obtener de los registros de cáncer.

Ante el problema de salud pública que constituye el cáncer, la Secretaría de Salud Federal promovió reformas a la Ley General de Salud, que fueron apoyadas por el

\section{Correspondencia:}

*Alejandro Mohar-Betancourt

E-mail: alejandro.mohar@salud.gob.mx
Date of reception: 01-02-2019

Date of acceptance: 28-02-2019

DOI: 10.24875/HMCM.M19000008 
Tabla 1. Cobertura poblacional y año de inicio de la Red

\begin{tabular}{llc}
\hline Ciudad/Estado & Año & Población (habs.) \\
\hline Mérida, Yucatán & 2016 & 777,615 \\
Guadalajara, Jalisco & 2017 & $5,035,880$ \\
Tijuana, Baja California & 2018 & $1,723,456$ \\
Campeche, Campeche & 2018 & 283,025 \\
Acapulco, Guerrero & 2018 & 789,971 \\
Toluca, Estado de México & 2018 & $1,101,363$ \\
& & $9,711,310$ \\
\hline
\end{tabular}

Tabla 2. Cobertura programada por la Red en 2018

\begin{tabular}{lc}
\hline Ciudad/Estado & Población (habs.) \\
\hline La Paz, Baja California Sur & 244,219 \\
Hermosillo, Sonora & 884,273 \\
León, Guanajuato & $1,578,626$ \\
Monterrey, Nuevo León & $1,109,171$ \\
& $3,816,289$ \\
\hline
\end{tabular}

Congreso de la Unión con la finalidad de crear en México la Red de RCBP. El objetivo es definir políticas públicas de prevención y tratamiento de la enfermedad, como resultado de la concentración de información relacionada con el número total de pacientes, tipos de cáncer, distribución por edad y sexo, diagnóstico, tratamiento y supervivencia y, con ello, dirigir la asignación de recursos y programas de prevención para su óptimo control.

Por tal motivo, ambas cámaras legislativas aprobaron la reforma de la Ley General de Salud, adicionando el artículo 161 Bis «Del Registro Nacional de Cáncer»5; mientras que el mes de julio del 2018 se publicó el Reglamento del Registro Nacional de Cáncer en el Diario Oficial de la Federación ${ }^{6}$.

\section{EL OBJETIVO}

El Registro Nacional tiene como objetivo la integración de una base de datos sobre los casos de cáncer en México. Proveer información consistente, completa, oportuna y de calidad, que sea útil para conformar, actualizar y analizar el panorama general de esta enfermedad; fomentar y desarrollar la investigación, la canalización de recursos, así como la formulación y evaluación de políticas públicas en la materia. Tiene como finalidades contar con información válida, fiable y comparable internacionalmente, que contribuya al conocimiento de la magnitud y distribución del cáncer en nuestro país, así como al estudio de sus causas y ser fuente oficial para la estimación de indicadores en los diversos programas de prevención, diagnóstico, tratamiento y cuidados paliativos que se desarrollen en México.

Contar con estos datos nos orientan para conocer la magnitud del problema del cáncer en nuestro país, ya que solo tenemos estimaciones desarrolladas por organismos internacionales como el GLOBOCAN ${ }^{1}$. Estas proyecciones incluyen generalmente la suposición de que las tendencias anteriores de incidencia y mortalidad se mantendrán, pero en ocasiones estas proyecciones subestiman la magnitud real de esta epidemia en México. Es por ello indispensable contar con una Red de RCBP representativa de la población mexicana.

\section{EL REGISTRO DE CÁNCER DE BASE POBLACIONAL EN MÉXICO}

A la fecha la Red cubre 9,711,310 habitantes de nuestro país. Ello representa el $8.12 \%$ de la población mexicana. La distribución por ciudad de esta Red y año de inicio de actividades es la se puede observar en la tabla 1.

Este año (2019) se iniciarán actividades en las ciudades que se observan en la tabla 2. 
La Red de RCBP tiene un sistema electrónico, REDCANCER MX, para la recolección de casos, el cual fue diseñado por la coordinación general del Registro Nacional de Cáncer. Dicho sistema permite la validación, análisis de información automatizada con datos codificados y estructurados que se apega a catálogos vigentes y permite la generación de reportes en tiempo real. Además, cumple con las validaciones internacionales de las siguientes instituciones: International Agency for Research in Cancer (OMS), Data Quality Checks (European Network of Cancer Registries).

A la fecha, se han registrado en esta Red 7,093 casos de cáncer. Ello representa el primer paso en la optimización de los recursos económicos para las enfermedades oncológicas, los servicios médicos e infraestructura necesarios y, con ello, mejorar el control del cáncer en
México, además de constituir un posible modelo para la vigilancia epidemiológica de otras patologías cronicodegenerativas, hoy de alta prevalencia en nuestro país.

\section{BIBLIOGRAFÍA}

1. Bray F, Ferlay J, Soerjomataram I, Siegel RL, Torre LA, Jemal A. Global cancer statistics 2018: GLOBOCAN estimates of incidence and mortality worldwide for 36 cancers in 185 countries. CA Cancer J Clin. 2018;68:394-424.

2. Haenszel W, Curnen MG. The first fifty years of the Connecticut Tumor Registry: reminiscences and prospects. Yale J Biol Med. 1986;59(5): 475-84.

3. National Cancer Institute. Surveillance, Epidemiology and End Results Program. Connecticut Registry [Internet]. U.S. Department of Health and Human Services, National Institutes of Health, National Cancer Institute; 2019. Disponible en: https://seer.cancer.gov/registries/connecticut.html

4. Cancer Incidence in Five Continents [Internet]. Lyon: International Agency for Research on Cancer; 2019. Disponible en: http://ci5.iarc.fr

5. Decreto por el que se reforman y adicionan diversas disposiciones de la Ley General de Salud. Diario Oficial de la Federación, 22 de junio 2017. México.

6. Reglamento del Registro Nacional de Cáncer. Diario Oficial de la Federación, 17 de julio 2018, México. 